\title{
BMJ Open Randomised, non-inferiority, controlled procedural outcomes TrIal comParing reverse T And Protrusion versus double- kissing and crush stenting: protocol of the TIP TAP I randomised trial
}

\author{
Kudrat Rakhimov (D) , ${ }^{1}$ Andrea Buono, ${ }^{1}$ Remzi Anadol, ${ }^{1,2}$ Helen Ullrich, ${ }^{1}$ \\ Maike Knorr, ${ }^{1}$ Majid Ahoopai, ${ }^{1}$ Thomas Münzel, ${ }^{1,2}$ Tommaso Gori ${ }^{1,2}$
}

To cite: Rakhimov K, Buono A, Anadol $\mathrm{R}$, et al. Randomised, non-inferiority, controlled procedural outcomes Trlal comParing reverse $\mathrm{T}$ And Protrusion versus doublekissing and crush stenting: protocol of the TIP TAP I randomised trial. BMJ Open 2020;10:e034264. doi:10.1136/ bmjopen-2019-034264

- Prepublication history for this paper is available online To view these files, please visit the journal online (http://dx.doi org/10.1136/bmjopen-2019034264).

Received 16 September 2019 Revised 29 January 2020 Accepted 13 February 2020

Check for updates

(c) Author(s) (or their employer(s)) 2020. Re-use permitted under CC BY-NC. No commercial re-use. See rights and permissions. Published by BMJ.

${ }^{1}$ Kardiologie I,

Universitätsmedizin Mainz,

Mainz, Rheinland-Pfalz,

Germany

${ }^{2}$ DZHK, Standort Rhein-Mainz, Universitätsmedizin Mainz,

Mainz, Rheinland-Pfalz,

Germany

Correspondence to Professor Tommaso Gori; tommaso.gori@unimedizinmainz.de

\section{ABSTRACT}

Introduction To assess the impact of 'reverse $T$ and Protrusion' (TAP) technique on the outcome after stenting of true bifurcation lesions of the left main (LM) or proximal epicardial vessels as compared with double kissing (DK)crush technique.

Methods and analysis 50 consecutive patients with true coronary bifurcation lesion (Medina 1,1,1 or $0,1,1$ ) of the LM or the proximal main vessels, requiring a twostent technique as first-line strategy at University Medical Center Mainz, are randomised in a 1:1 ratio to reverse TAP or DK-crush stenting. As recommended by best clinical practice, final angiographic result is evaluated and optical coherence tomographic (OCT) intracoronary imaging is performed to assess and optimise the final result. The primary end point is defined as the percentage of stent expansion in the side branch. Secondary end points consist of angiographic and procedural success (assessed until patient's discharge), procedural parameters (procedural time, fluoroscopy time, use of devices, $\mathrm{X}$-ray dose) and OCT parameters expressing expansion of the stents. Safety parameters include all adverse events up to 6 months after discharge. A clinical, angiographic and intracoronary imaging control at 6 months is planned. Ethics and dissemination The protocol complies with good clinical practice and the ethical principles described in the Declaration of Helsinki and is approved by the local ethics committee. The results of the trial will be published as original article(s) in medical journals and/or as presentation at congresses.

Trial registration number ClinicalTrials.gov Registry (NCT03714750)

\section{BACKGROUND}

Coronary bifurcations are a typical challenge of percutaneous coronary interventions (PCI).

With the introduction of drug eluting stents, the long-term outcome of these interventions has dramatically improved, justifying also the use of double-stent techniques, which in the era of bare metal stents were

\section{Strength and limitations of this study}

- This is the first study comparing the effect of a new two-stent technique called reverse $\mathrm{T}$ and Protrusion with the standard treatment (double kissing crush) for bifurcation coronary lesions.

- Allocation is performed in a randomised fashion.

- Angiographic assessment is implemented by poststenting optical coherence tomographic (OCT) imaging to maximally optimise the final result.

- This is a small study with an insufficient sample size to address clinical end points, such as mortality and myocardial infarction.

- Blinding at the time of treatment is not feasible; however, OCT imaging will be analysed by blinded personnel.

prone to an unacceptably high risk of restenosis. ${ }^{1-3}$ An important role in bifurcation treatment is played by the technique used for implanting the stents. Provisional stenting (PS) is the simplest and most adopted technique for bifurcations, and it can be described as stenting in the main branch (MB) and only balloon angioplasty (percutaneous coronary angioplasty (PTCA)) of the side branch $(\mathrm{SB})$. In this approach, SB stenting is performed only if the result at the ostium is suboptimal after PTCA, as defined by thrombolysis in myocardial infarction flow $<3$ or greater than type $\mathrm{B}$ dissection or a higher grade residual stenosis. In the EXCEL (Evaluation of XIENCE versus Coronary Artery Bypass Surgery for Effectiveness of Left Main Revascularization) trial, which randomised 1905 patients with unprotected left main (LM) disease and low or intermediate SYNTAX (Synergy Between Percutaneous Coronary Intervention With Taxus and Cardiac Surgery) scores to PCI with a 
second-generation everolimus-eluting stents versus coronary artery bypass grafting (CABG), most patients were treated with PS. ${ }^{4}$ The results showed that PCI with everolimus-eluting stents was non-inferior to CABG with respect to the composite end point of death, stroke or myocardial infarction at 3 years, superior with regards to major events (eg, arrhythmias and bleeding) but inferior in terms of ischaemia-driven revascularisation during follow-up (in $12.6 \%$ vs $7.5 \%$ of the patients, $\mathrm{p}<0.001$ ), which was probably due to the stenting technique used. Moreover, PS appears to be not effective when the SB is $>2.5 \mathrm{~mm}$, with $>50 \%$ stenosis extending $>5 \mathrm{~mm}$ beyond the ostium. In this case, a two-stent technique is usually considered. Two-stent techniques are more complex, and the literature offers a wide range of theoretical options as summarised by the Main, Across, Distal, Side classification. ${ }^{5}$ Of these, the one with the most promising results appear to be the so-called double kissing (DK)-crush technique, which consists of the following steps: stenting the $\mathrm{SB}$ (with $1-2 \mathrm{~mm}$ protrusion in the $\mathrm{MB}$ ), $\mathrm{SB}$ stent balloon crush (with a balloon placed in the MB), proximal rewiring of SB access through the crushed stent, first kissing balloon inflation (KBI), MB stenting across the SB after SB wire is removed, proximal optimising technique (POT), second SB rewiring (through the MB stent), second KBI and final re-POT. ${ }^{6}$ In the DK-crush V trial, compared with PS, DK crush was superior in terms of target lesion failure (TLF) (HR: 0.42; 95\% CI: 0.21 to $0.85 ; \mathrm{p}=0.02)$, target vessel myocardial infarction $(2.9 \%$ vs $0.4 \% ; \mathrm{p}=0.03$ ) and definite or probable stent thrombosis (ST) $(3.3 \%$ vs $0.4 \% ; \mathrm{p}=0.02){ }^{8}$

For non-LM lesions, PS has been shown to be noninferior or even superior to a planned two-stent approach consisting of T-stenting, crush or culotte stenting in most randomised trials. ${ }^{9}{ }^{10}$ In contrast, the DK crush (also double-stent, but resulting in better lesion coverage) resulted in lower rates of target lesion revascularisation (TLR) compared with PS in non-LM coronary bifurcation lesions. ${ }^{11}$ In the DK crush II study, at 5 years, the major adverse cardiac event rate $(23.8 \%)$ in the PS group was insignificantly different to that of the DK-crush group (15.7\%; $\mathrm{p}=0.051)$. However, the difference in the TLR rate between two groups was sustained through the 5-year follow-up ( $16.2 \%$ vs $8.6 \%$; $\mathrm{p}=0.027) .{ }^{12}$ As compared with traditional two-stent techniques, DK crush is superior in terms of ST and composite major adverse cardiac events. $^{13} 14$ Moreover, particular attention has to be focused on unprotected LM bifurcation lesions, since these patients present a significantly higher daily risk of events after PCI, ${ }^{15}$ and an optimal stenting strategy is crucial to guarantee the best clinical result.

Based on the above evidence, although a trial comparing DK crush with CABG has not yet been performed, DK crush is considered to be the gold standard for bifurcation stenting. DK crush has however important limitations: first, it requires two rewiring procedures. This procedure can be complex, may require time and materials, involves elevated X-ray exposure and potentially exposes the patient to the complications associated with prolonged manipulation, including a risk of intraprocedural thrombosis, vessel dissection and a higher dose of contrast medium. Second, it still involves crushing of the SB stent, resulting in three layers of metal at the level of the bifurcation. To date, no data on the reverse $\mathrm{T}$ and Protrusion (TAP) technique (a modified two-stent technique) are available in literature.

The study being proposed therefore will assess the impact of a modified stenting technique (called reverse TAP) on the outcome after stenting of true bifurcation lesions (Medina type $1,1,1$ or $0,1,1$ ) of the LM or proximal epicardial vessels. Patients enrolled are randomly allocated to either a DK-crush or modified reverse TAP strategy.

\section{Aim of the study}

The aim of this study is to assess the impact of reverse TAP on the outcome after stenting of true bifurcation lesions (Medina type $1,1,1$ or $0,1,1$ ) of the LM or proximal epicardial vessels (left anterior descending coronary artery (LAD) of left circumflex artery (LCX)). Results are compared with the standard technique for bifurcation treatment, namely DK crush. These findings may provide new recommendation on which two-stent technique is associated with the best result.

\section{METHODS/DESIGN}

This study is a single-centre, randomised equivalence trial to compare two-stent strategies for the treatment of coronary bifurcation lesions, conducted in the catheterisation laboratory of the Kardiologie I, University Medical Center Mainz, Germany. Expansion to two other laboratories is planned.

Fifty consecutive patients with LM or LAD/LCX coronary bifurcation lesions with an indication to percutaneous revascularisation (both acute and elective presentations) are assigned in a 1:1 ratio to one of the two two-stent technique described below.

Patients must meet all of the inclusion criteria: age $\geq 18$; ability to give informed consent, documented heart team (as per guidelines) decision for revascularisation via PCI; planned PCI for a bifurcation stenosis with both branches $>2.5 \mathrm{~mm}$, but $\leq 5.0 \mathrm{~mm}$, and with a stenosis $>50 \%$ and indication to revascularisation including ischaemic symptoms, positive non-invasive imaging for ischaemia or positive fractional flow reserve (FFR) $<0.8$ or minimal lumpen area $<6 \mathrm{~mm}^{2}$ for the $\mathrm{LM}$ or $<4 \mathrm{~mm}^{2}$ for epicardial vessels; true bifurcation lesion Medina type $1,1,1$ or $0,1,1$.

Patients will be excluded if any of the following criteria applies: cardiogenic shock as clinical presentation; trifurcation if all vessels are $\geq 2.75 \mathrm{~mm}$ diameter; either bifurcation vessel not suitable for stenting; history of stenting in target bifurcation lesion; participation in another investigational drug or device study; women of childbearing potential or lactating and patient unable to give informed consent. 


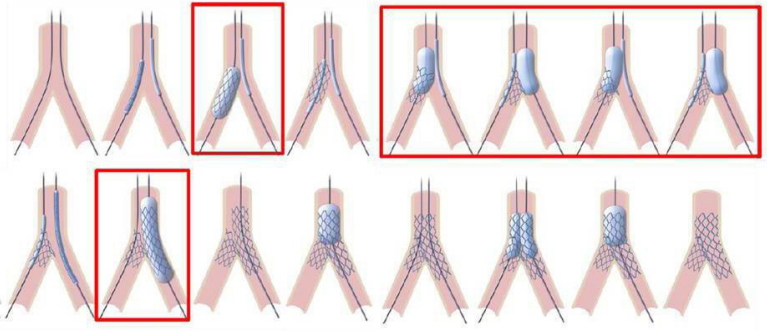

Figure 1 Steps of reverse TAP technique. (A) SB and MB wiring; (B-D) SB stenting; (E) sequential balloon inflation in SB and MB; (F, G) MB stenting; $(\mathrm{H})$ SB wire removal; (I) MB POT; (J) SB rewiring; (K) KBI; (L) final POT and (M) final result. The main differences compared with DK crush are highlighted with red boxes and consist of the minimal protrusion of SB stent in $\mathrm{MB}$, avoiding a double layer of struts in the proximal $\mathrm{MB}$ (in $\mathrm{C}$ ), sequential balloon inflation in $\mathrm{SB}$ and $\mathrm{MB}$ (in $\mathrm{E}$ ) instead of the first $\mathrm{KBI}$ in $\mathrm{DK}$ crush and the possibility to keep in position the SB wire while MB stenting (in $\mathrm{G}$ ) with the need of only one SB rewire during the entire procedure (in $\mathrm{G}$ ) compared with the two SB rewiring requested in DK crush. $\mathrm{DK}$, double kissing; $\mathrm{KBI}$, kissing balloon inflation; $\mathrm{MB}$, main branch; POT, proximal optimising technique; reverse TAP, reverse $T$ and Protrusion; SB, side branch;

After stenting of $\mathrm{MB}$ and $\mathrm{SB}$, final result is assessed by optical coherence tomography (OCT) imaging.

All end points that are relevant for this study are collected at the time of the procedure and at the time of patient's discharge. As per clinical routine, most patients undergo a clinical visit and angiography, including intracoronary imaging, at 6 months after stenting. These data will be collected as additional information on the occurrence of complications after the procedure only for the purpose of safety.

The SPIRIT checklist ${ }^{16}$ for reporting a clinical trial was used. Data acquisition started in October 2018. The duration of this study is expected to be 18 months.

\section{Reverse TAP technique}

A scheme of the reverse TAP is shown in figure 1 with the most relevant differences as compared with DK crush emphasised with red boxes.

Reverse TAP technique adopted in this study requires in sequence the following steps:

1. Wiring of both $\mathrm{SB}$ and $\mathrm{MB}$ (figure $1 \mathrm{~A}$ ).

2. SB stenting with minimal protrusion of the stent in the MB (figure 1B-D). As such, the amount of crush is minimised (avoiding crush of the entire SB stent on the lateral wall of the MB proximal to the bifurcation). This limits the amount of protruding metal, a potential source of ST. Also, the absence of complete crushing allows continuing the procedure without having to perform an additional wiring. This could be another advantage of the technique since rewiring may be sometimes complicated (or impossible), translating in increased need of contrast medium, procedural time and materials. ${ }^{17}$

3. Sequential balloon inflation in $\mathrm{SB}$ and $\mathrm{MB}$ with final minimal crush of SB stent (figure 1E). The sequential balloon inflations allow the homogeneous distribution of the struts along the circumference of the ostium, which again limits the amount of metal in the MB. Also, the sequential dilation reduces the chance that the MB stent remains trapped in the SB stent when being advanced. ${ }^{17}$ The absence of complete crush also allows maintaining the SB wire until after implantation of the MB stent. This improves the chance of patency of the SB.

4. MB stenting, without removing the SB wire (figure $1 \mathrm{~F}, \mathrm{G})$.

5. SB wire removal and POT of MB (figure $1 \mathrm{H}, \mathrm{I}$ ).

6. SB rewiring and $\mathrm{KBI}$ (figure $1 \mathrm{~J}, \mathrm{~K}$ ).

7. Final POT (figure 1L).

\section{DK-crush technique}

As previously described (see the Background section).

\section{Study setting}

The University Medical Center Mainz is a high-volume tertiary clinic. Experienced interventional cardiologists perform invasive exams. OCT images are assessed by experienced cardiologists and fellows.

\section{Study intervention}

Investigation medical product

Patients will be randomised to one of two groups:

1. Reverse TAP technique (experimental group).

2. DK-crush technique (control group).

All patients will undergo OCT imaging after bifurcation stenting.

A scheme of this study is presented in figure 2 .

For this study, the following two-stent types will be used exclusively, both having a CE mark:

1. XIENCE PRO Everolimus-eluting Coronary and Peripheral Stent Systems, Abbott Vascular, Santa Clara California, CE no 632827.

2. CoroflexISAR and CoroflexISAR Neo by B. Braun Melsungen AG, Melsungen, Germany, CE no 39.05.657.

OCT catheter used is OPTIS DragonFly Abbott Vascular, Santa Clara, USA.

\section{Study end points}

Primary end point

1. The primary end point is the percentage of stent expansion in the SB as assessed by OCT. Stent expansion is described as the ratio of the minimum stent area of the SB and the maximum stent area of the SB. Ostial $\mathrm{SB}$ is defined as the proximal $3 \mathrm{~mm}$ segment of the vessel.

Since OCT analysis will be performed offline, the primary end point per se will not be a reason for further treatment during the index procedure.

\section{Secondary end points}

Secondary outcomes include:

1. Fluoroscopy and procedural time.

2. Number of coronary wires used. 


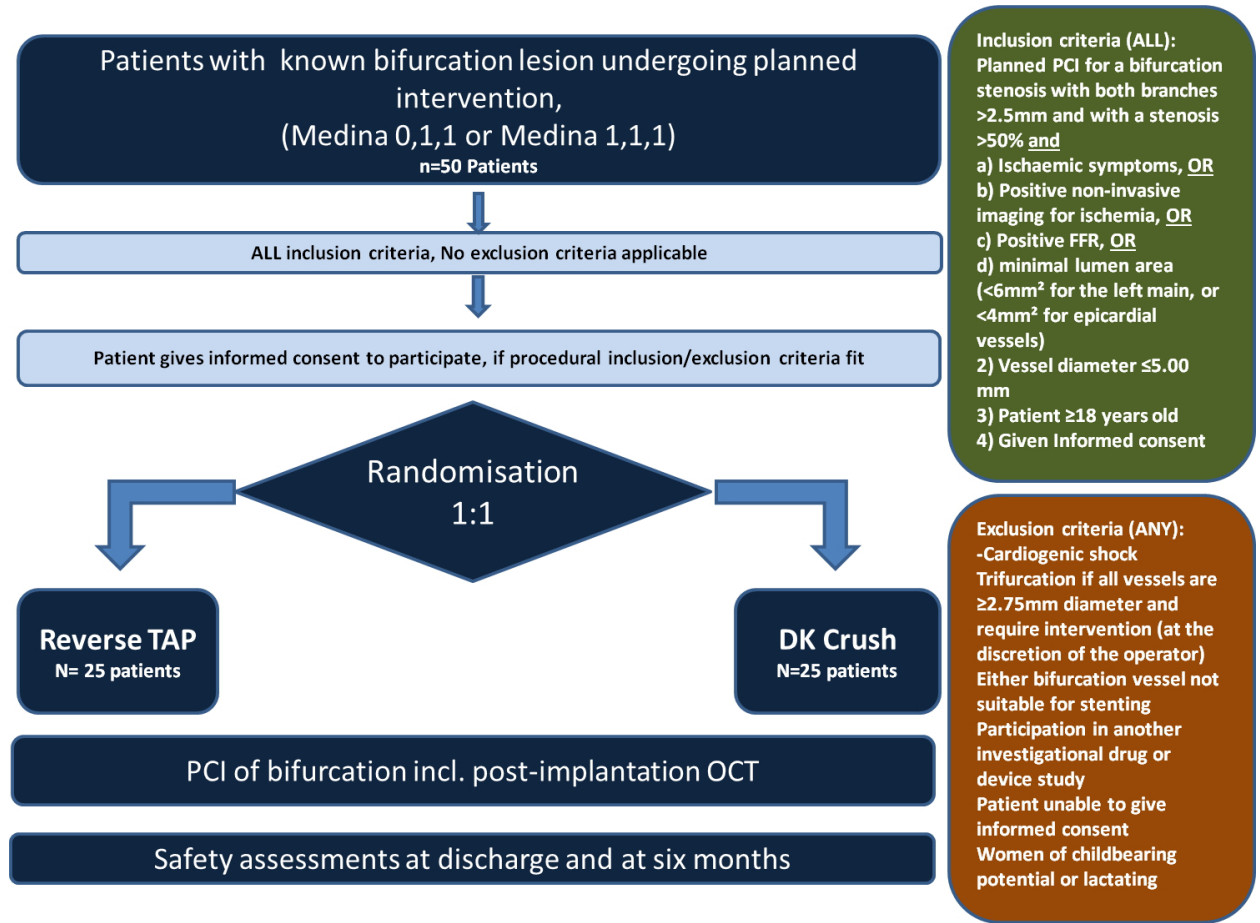

Figure 2 Overview of the study. DK, double kissing; FFR, fractional flow reserve; OCT, optical coherence tomography; PCI, percutaneous coronary interventions; reverse TAP, reverse T and Protrusion.

3. Protocol success (defined if intervention is performed according to the protocol, including final KBI).

4. Minimum lumen diameter and percentage of stenosis diameter in the $\mathrm{MB}$ and the $\mathrm{SB}$, assessed at quantitative coronary angiography (QCA) analysis.

5. Procedural success as defined by angiographic success (no residual stenosis $>20 \%$ at the end of the intervention) and no periprocedural complications including ST elevation myocardial infarction, new Q-wave myocardial infarction, death, ST, CABG or periprocedural cardiac biomarker release at discharge.

6. OCT end points (all measured in the $\mathrm{MB}$ and $\mathrm{SB}$ ): average Stent Eccentricity Index, calculated as the ratio of the minimum to maximum stent diameter; minimum and mean lumen diameter; minimum and mean lumen area.

\section{Analysis of OCT data}

OCT data are analysed offline using QCU-CMS V.4-69 (Leiden University Medical Center in cooperation with MEDIS, Leiden). Stented segments each with $0.5 \mathrm{~mm}$ through the entire length are assessed, measuring manually diameters and areas.

\section{Randomisation}

Consecutive patients are randomised. Randomisation is done in a 1:1 ratio using blocks (each block 10 exams) without stratification. Randomisation is done by using a computer-generated random sequence (Medcalc, Mariakerke, Belgium). Randomisation is performed during interventional procedure, after wiring both MB and SB. In case bifurcation treatment is not feasible at all or according to randomisation indicated technique, patient is excluded from this study and is considered a screening failure.

\section{Selection and withdrawal \\ Recruitment}

Patient recruitment is performed within the patients treated at the Kardiologie I of the Universitätsmedizin Mainz. Patients planned (based on clinical indication and current guidelines, including a documented heart team consensus) for PCI of the LM or an LM-equivalent lesion will be asked for interest in study participation. Consent will be obtained by study physicians before beginning of the procedure.

\section{Withdrawal criteria}

Patients can withdraw from this study at any time.

In case bifurcation treatment is not feasible with one or both of the study techniques, the patient is excluded from this study and is considered a screening failure.

\section{Informed consent}

All patients will sign a consent in which the nature of the experimental and control groups is clearly explained. All patient data are pseudonymised and collected by this study team. Since all procedures belong to clinical standards, there are no additional risks/implications connected with the participation in this study.

\section{Statistics}

Statistical analysis will be performed with $\mathrm{R}$ and Medcalc (Mariakerke, Belgium).

OCT and QCA parameters will be compared after final kissing balloon using analysis of covariance using the 
absolute vessel diameter as covariate. Significance will be determined after multiple pairwise comparisons with the Bonferroni post-hoc test. Data will be presented as mean \pm SD or median (IQR); a p value $<0.05$ will be considered statistically significant for the primary end point.

For the secondary procedural end points, the MannWhitney $\mathrm{U}$ test will be used. All secondary end points will be analysed by descriptive statistics and appropriate exploratory $\mathrm{p}$ values.

\section{Power calculation}

The power calculation is based on the data of comparing traditional crush with DK crush. ${ }^{18}$ Primary end point of this study is percentage of stent expansion in the SB. The study by Shan $e t a l^{18}$ reported a $72 \%$ expansion in the DK crush as compared with 53\% in the traditional crush group with SD expansion rates of $11.5 \%$ and $13.5 \%$, respectively. Our study is designed as a continuous outcome equivalence trial assuming an SD of $11 \%$ and equivalence limit of $11 \%$, that is, $50 \%$ of the advantage of DK crush versus traditional crush. If there is no difference between the standard (DK crush) and experimental arm, then 44 patients would be required for a power of $90 \%$ to exclude a difference in means of more than $11 \%$, given a two-sided CI of $90 \%$. Assuming an attrition (including impossibility to cross the bifurcation with the OCT catheter) of $\sim 15 \%$, we planned to recruit 50 patients.

\section{Analysis populations}

All procedures that are assigned a randomisation number are considered as enrolled/randomised procedures.

This study, which is not designed or powered to test clinical end points, has an OCT end point as primary end point. The following events will however be recorded, as they represent the standard clinical end points that are assessed to determine the safety and outcome after interventional procedures. All clinical data will be collected at discharge from hospital and at the 6-month clinical visit, which is recommended to all patients who underwent intervention of complex lesions and a standard procedure in patients undergoing LM or complex bifurcation stenting.

\section{Efficacy analyses}

The primary analysis will be on the per-protocol principle (ie, including all patients who are not protocol violators). A separate analysis will be performed on an intention-totreat basis (ie, all randomised patients randomised to a treatment arm).

\section{Safety analyses}

The procedures related to this study do not interfere with normal routine in the catheterisation laboratory. Risks are linked with any of the two intracoronary procedures and a difference between the two groups is not expected.

Periprocedural cardiac biomarker release is defined as ${ }^{19}$ :

1. In patients undergoing PCI with normal ( $\leq 99$ th percentile URL) baseline cardiac troponin (cTn) concentrations, elevations of cTn $>5$ times the 99th percentile URL occurring within 48 hours of the procedure.

2. In patients undergoing PCI with elevated baseline, $\mathrm{cTn}$ values and two measures are available showing stable or falling values, a rise of $>20 \%$ after PCI.

3. In patients with elevated cTn levels before PCI and raising $\mathrm{cTn}$ or only one $\mathrm{cTn}$ value available, this end point cannot be evaluated.

Other safety end points to be analysed at discharge and at 6 months (standard planned clinical interview):

1. TLF, defined as a composite of cardiovascular death, target-vessel myocardial infarction.

2. TLR: any (including attempted) repeat revascularisation with either balloon angioplasty or stenting, or CABG, within the previous treated vessel segment including the $5 \mathrm{~mm}$ proximal or distal. Where there is doubt about the need for reintervention, physicians are strongly recommended to use FFR of invasive imaging to ascertain whether reintervention is required.

3. Death: cause of death should be recorded. Cause of death will be considered cardiac unless specified otherwise. In the primary analysis, all deaths will be compared. The coordinating site must be notified of a patient's death within 3 days of its knowledge. Data related to the patient's death should be recorded. A copy of the death certificate with anonymised study identification number but with the patient's name removed should be sent to the coordinating site within 3 weeks of the patient's death. Postmortem results if available should be followed as soon as possible.

4. Cardiac death: any death due to proximate cardiac cause (eg, myocardial infarction, low-output failure, fatal arrhythmia), unwitnessed death and death of unknown cause and all procedure-related deaths, including those related to concomitant treatment.

5. Vascular death: death caused by non-coronary vascular causes, such as cerebrovascular disease, pulmonary embolism, ruptured aortic aneurysm, dissecting aneurysm or other vascular diseases.

6. Non-cardiovascular death: any death not covered by the above definitions, such as death caused by infection, malignancy, sepsis, pulmonary causes, accident, suicide or trauma.

7. Clinically relevant myocardial infarction. The Universal definition of Myocardial Infarction (Revision 2013) will be used to define clinically relevant myocardial infarction in this study. ${ }^{20}$

Periprocedural myocardial infarction is defined as: in patients with normal baseline creatinin-phosphokinase (CK-MB), the peak CK-MB measured within 48 hours of the procedure rises to $\geq 10$ times the local laboratory upper limit of normality (ULN), or to $\geq 5$ times ULN with new pathological Q-waves in $\geq 2$ contiguous leads or new persistent left bundle branch block (LBBB), or in the absence of CK-MB measurements and a normal baseline cTn, a cTn (I or T) level measured within 48 hours of the PCI rises to $\geq 70$ times the local laboratory ULN, or $\geq 35$ 
times ULN with new pathologic Q-waves in $\geq 2$ contiguous leads or new persistent LBBB; in patients with elevated baseline CK-MB (or cTn) in whom the biomarker levels are stable or falling, the CK-MB (or cTn) rises by an absolute increment equal to those levels recommended above from the most recent preprocedure level and in patients with elevated CK-MB (or cTn) in whom the biomarker levels have not been shown to be stable or falling, the CK-MB (or cTn) rises by an absolute increment equal to those levels recommended above plus new ST-segment elevation or depression plus signs consistent with a clinically relevant myocardial infarction (MI), such as new onset, worsening heart failure or sustained hypotension.

\section{Confidentiality}

Patient data will be pseudonymised and collected by this study team. Pseudonymised patient data will be stored digitally on an external hard drive not connected to the clinical intranet and accessible only to the members of this study team. After 10 years of storage, data will be destroyed. It is not intended to give study participants' data to a third party. All data will be analysed after the last patient is discharged from index hospitalisation. No interim analysis is intended. However, a Data Safety Monitoring Board consisting of two physicians not affiliated with this study will monitor the safety of the subjects throughout this study. In case a study participant withdraws consent after having his data collected from him, the patient's data will be anonymised.

\section{Patient and public involvement}

No patient involved in the design of this study.

\section{Ethics and dissemination}

The protocol was approved by the ethics committee of the local Landesärztekammer (2018-13441-KliFo). The procedures set out in this trial protocol, pertaining to the conduct, evaluation and documentation of this trial, are designed to ensure that all persons involved in the trial abide by good clinical practice (GCP) and the ethical principles described in the Declaration of Helsinki. The trial is carried out in keeping with local legal and regulatory requirements. The requirements of the Arzneimittelgesetz, the GCP regulation and the Federal Data Protection Law are kept.

Publication resulting from this study will require the approval of the co-authors. The results of the trial will be published as original article(s) in medical journals and/or as presentation at congresses. The principal investigator is senior author of this article. He or his delegates will present the data at appropriate congresses. The choice of the journal for the publication will be made by the principal investigator in agreement with the co-authors. Besides the principal investigator, a substantial contribution to the recruitment of subjects, to interpretation of the data and/or to drafting the article or revising it qualifies for authorship. Source data will be made available on justified request.

\section{Audit}

Competent authorities and sponsor authorised persons (auditor) may request access to all source documents, CRF and other trial documentation in case of an inspection or audit. Direct access to these documents will be guaranteed by the principal investigator who will provide support at all times for these activities. Source data documents can be copied during inspection or audit after the identity of the subject have been made unrecognisable. No external audits are planned for this trial.

\section{Insurance}

Since all procedures (except for randomisation) are clinically indicated and acknowledged in the current literature, study insurance is not planned.

\section{Study committees}

The Clinical Event Committee consists of two physicians blinded to the allocation group who will assess all clinical events, including Safety Adverse Events.

The Data Safety Monitoring Board consists of two physicians and a biostatistician who will monitor the safety of the subjects throughout this study.

\section{Trial status}

Data acquisition is ongoing. The first patient was included in October 2018. We expect the study to be closed in December 2020.

Contributors RA and TG have provided intellectual input for the design and planning of the study. The manuscript and protocol were initially written by RA and $\mathrm{TG}$, and they were corrected and edited by KR, $A B$ and TG. All authors (KR, $A B, R A$, $\mathrm{HU}, \mathrm{MK}, \mathrm{MA}, \mathrm{TM}$ and TG) critically revised the final draft of the manuscript for its intellectual content and approved the final manuscript.

Funding The study will be financed by own means of the Center of Cardiology of the University Hospital Mainz (=Sponsor) and means of the W3-Professorship of translational myocardial and cardiovascular function. No third-party funds are planned.

Competing interests The trial is funded by the Kardiologie I, University Medical Center Mainz. No manufacturer of the drugs has been involved in this study. None of the authors has conflicts of interest to declare.

Patient and public involvement Patients and/or the public were not involved in the design, or conduct, or reporting, or dissemination plans of this research.

Patient consent for publication Not required.

Provenance and peer review Not commissioned; externally peer reviewed.

Open access This is an open access article distributed in accordance with the Creative Commons Attribution Non Commercial (CC BY-NC 4.0) license, which permits others to distribute, remix, adapt, build upon this work non-commercially, and license their derivative works on different terms, provided the original work is properly cited, appropriate credit is given, any changes made indicated, and the use is non-commercial. See: http://creativecommons.org/licenses/by-nc/4.0/.

ORCID iD

Kudrat Rakhimov http://orcid.org/0000-0003-3936-8753

\section{REFERENCES}

1 Morice M-C, Serruys PW, Kappetein AP, et al. Five-year outcomes in patients with left main disease treated with either percutaneous coronary intervention or coronary artery bypass grafting in the synergy between percutaneous coronary intervention with taxus and cardiac surgery trial. Circulation 2014;129:2388-94. 
2 Morice M-C, Feldman TEE, Mack MJ, et al. Angiographic outcomes following stenting or coronary artery bypass surgery of the left main coronary artery: fifteen-month outcomes from the synergy between $\mathrm{PCl}$ with TAXUS express and cardiac surgery left main angiographic substudy (SYNTAX-LE MANS). Eurolntervention 2011;7:670-9.

3 Serruys PW, Morice M-C, Kappetein AP, et al. Percutaneous coronary intervention versus coronary-artery bypass grafting for severe coronary artery disease. N Engl J Med 2009;360:961-72.

4 Stone GW, Sabik JF, Serruys PW, et al. Everolimus-Eluting stents or bypass surgery for left main coronary artery disease. $N$ Engl $\mathrm{J}$ Med 2016;375:2223-35.

5 Louvard Y, Thomas M, Dzavik V, et al. Classification of coronary artery bifurcation lesions and treatments: time for a consensus! Cathet. Cardiovasc. Intervent. 2008;71:175-83.

6 Sawaya FJ, Lefèvre T, Chevalier B, et al. Contemporary approach to coronary bifurcation lesion treatment. JACC Cardiovasc Interv 2016;9:1861-78.

7 Zhang J-J, Chen S-L. Classic crush and DK crush stenting techniques. Eurolntervention 2015;11 Suppl V:V102-5.

8 Chen S-L, Zhang J-J, Han Y, et al. Double kissing crush versus provisional stenting for left main distal bifurcation lesions: DKCRUSH-V randomized trial. J Am Coll Cardiol 2017;70:2605-17.

9 Steigen TK, Maeng M, Wiseth R, et al. Randomized study on simple versus complex stenting of coronary artery bifurcation lesions: the nordic bifurcation study. Circulation 2006;114:1955-61.

10 Hildick-Smith D, de Belder AJ, Cooter N, et al. Randomized trial of simple versus complex drug-eluting stenting for bifurcation lesions: the British bifurcation coronary study: old, new, and evolving strategies. Circulation 2010;121:1235-43.

11 Chen S-L, Santoso T, Zhang J-J, et al. A randomized clinical study comparing double kissing crush with provisional stenting for treatment of coronary bifurcation lesions: results from the DKCRUSH-II (double kissing crush versus provisional stenting technique for treatment of coronary bifurcation lesions) trial. J Am Coll Cardiol 2011;57:914-20.

12 Chen S-L, Santoso T, Zhang J-J, et al. Clinical outcome of double kissing crush versus provisional stenting of coronary artery bifurcation lesions: the 5-year follow-up results from a randomized and multicenter DKCRUSH-II study (randomized study on double kissing crush technique versus provisional stenting technique for coronary artery bifurcation lesions). Circ Cardiovasc Interv 2017;10:e004497.

13 Chen S-L, Xu B, Han Y-L, et al. Comparison of double kissing crush versus Culotte stenting for unprotected distal left main bifurcation lesions: results from a multicenter, randomized, prospective DKCRUSH-III study. J Am Coll Cardiol 2013;61:1482-8.

14 Chen S-L, Xu B, Han Y-L, et al. Clinical outcome after DK crush versus culotte stenting of distal left main bifurcation lesions: the 3-year follow-up results of the DKCRUSH-III study. JACC Cardiovasc Interv 2015;8:1335-42.

15 Biolè C, Huczek Z, Nuñez-Gil I, et al. Daily risk of adverse outcomes in patients undergoing complex lesions revascularization: a subgroup analysis from the RAIN-CARDIOGROUP VII study (very thin stents for patients with left main or bifurcation in real life). Int $J$ Cardiol 2019;290:64-9.

16 Chan AW, Tetzlaff JM, Altman DG, et al. Statement: defining standard protocol items for clinical trials. Rev Panam Salud Publica 2013;2015:506-14.

17 Chen S, Tan H, Lee M, et al. More modified crush techniques for coronary bifurcation lesions: which one is better? Catheter Cardiovasc Interv 2007;69:468-9.

18 Shan S-J, Ye F, Liu Z-Z, et al. Coronary bifurcation lesions treated with double kissing crush technique compared to classical crush technique: serial intravascular ultrasound analysis. Chin Med J 2013;126:1247-51.

19 Moussa ID, Klein LW, Shah B, et al. Consideration of a new definition of clinically relevant myocardial infarction after coronary revascularization: an expert consensus document from the Society for cardiovascular angiography and interventions (SCAI). J Am Coll Cardiol 2013;62:1563-70.

20 Thygesen K, Alpert JS, Jaffe AS, et al. Third universal definition of myocardial infarction. Circulation 2012;126:2020-35. 